\title{
Synchronous ileal inflammatory fibroid polyp and Meckel's diverticulum found during laparoscopic surgery for adult intussusception
}

\author{
Sung II Kang ${ }^{1}$, Mi Jin Gu ${ }^{2}$ \\ ${ }^{1}$ Department of Surgery, Yeungnam University College of Medicine, Daegu, Korea \\ ${ }^{2}$ Department of Pathology, Yeungnam University College of Medicine, Daegu, Korea
}

Received: August 16, 2019

Revised: November 26, 2019

Accepted: December 8, 2019

Corresponding author:

Sung II Kang

Department of Surgery, Yeungnam

University College of Medicine, 170

Hyeonchung-ro, Nam-gu, Daegu

42415, Korea

Tel: +82-53-620-3580

Fax: +82-53-624-1213

E-mail:sungiry@naver.com
We present a rare case of synchronous ileal inflammatory fibroid polyp and Meckel's diverticulum detected during laparoscopic surgery for adult intussusception. A 48-year-old woman presented with sudden onset of severe abdominal pain. Abdominal computed tomography revealed a segment of ileocecal intussusception. Thus, laparoscopic exploration was performed, which revealed an ileal mass with an outpouching closed luminal structure in the distal ileum. Two abnormal structures were resected via mini-laparotomy, and the patient was discharged without postoperative complications. Histopathological examination confirmed an ileal inflammatory fibroid polyp and Meckel's diverticulum with ectopic pancreatic tissue.

Keywords: Intestinal obstruction; Intestinal polyps; Intussusception; Meckel diverticulum

\section{Introduction}

Intussusception rarely causes intestinal obstruction in adults and accounts for approximately $1 \%-5 \%$ of all cases of bowel obstruction [1]. In contrast to pediatric intussusception, most cases of adult intussusception are secondary to an underlying pathology, such as primary/metastatic malignancies or benign tumors that serve as the leading point of intussusception [2].

An inflammatory fibroid polyp (IFP) is a benign tumor usually localized to the gastric antrum, although it can occur throughout the gastrointestinal tract [3]. Meckel's diverticulum (MD) is a remnant of the omphalomesenteric duct occurring in approximately $2 \%$ of the population [4]. Both IFP and MD can act as leading points in adult intussusception. We present a rare case of synchronous ileal IFP and MD noted during laparoscopic surgery for adult intussusception.

\section{Case}

A 48-year-old woman was referred to our emergency department due to a sudden onset of severe abdominal pain. Her vital signs were stable on physical examination, and direct tenderness in the right lower quadrant without rigidity or distention was observed on abdominal examination. The patient reported a history of total laparoscopic hysterectomy for early-stage endometrial cancer, 4 years prior to the presentation. Abdominal radiography revealed a stepladder sign with several small bowel loops. Blood tests were within normal limits. Abdominal computed tomography (CT) revealed ileocecal intussusception with proximal small bowel obstruction (Fig. 1).

Urgent laparoscopic exploration was performed using 3 trocars (a 12-mm supraumbilical port for the camera and two 5-mm ports placed in the left middle and lower quadrants of the abdo-

Copyright (C) 2020 Yeungnam University College of Medicine

This is an Open Access article distributed under the terms of the Creative Commons Attribution Non-Commercial License (http://creativecommons.org/licenses/by-nc/4.0/) which permits unrestricted non-commercial use, distribution, and reproduction in any medium, provided the original work is properly cited. 

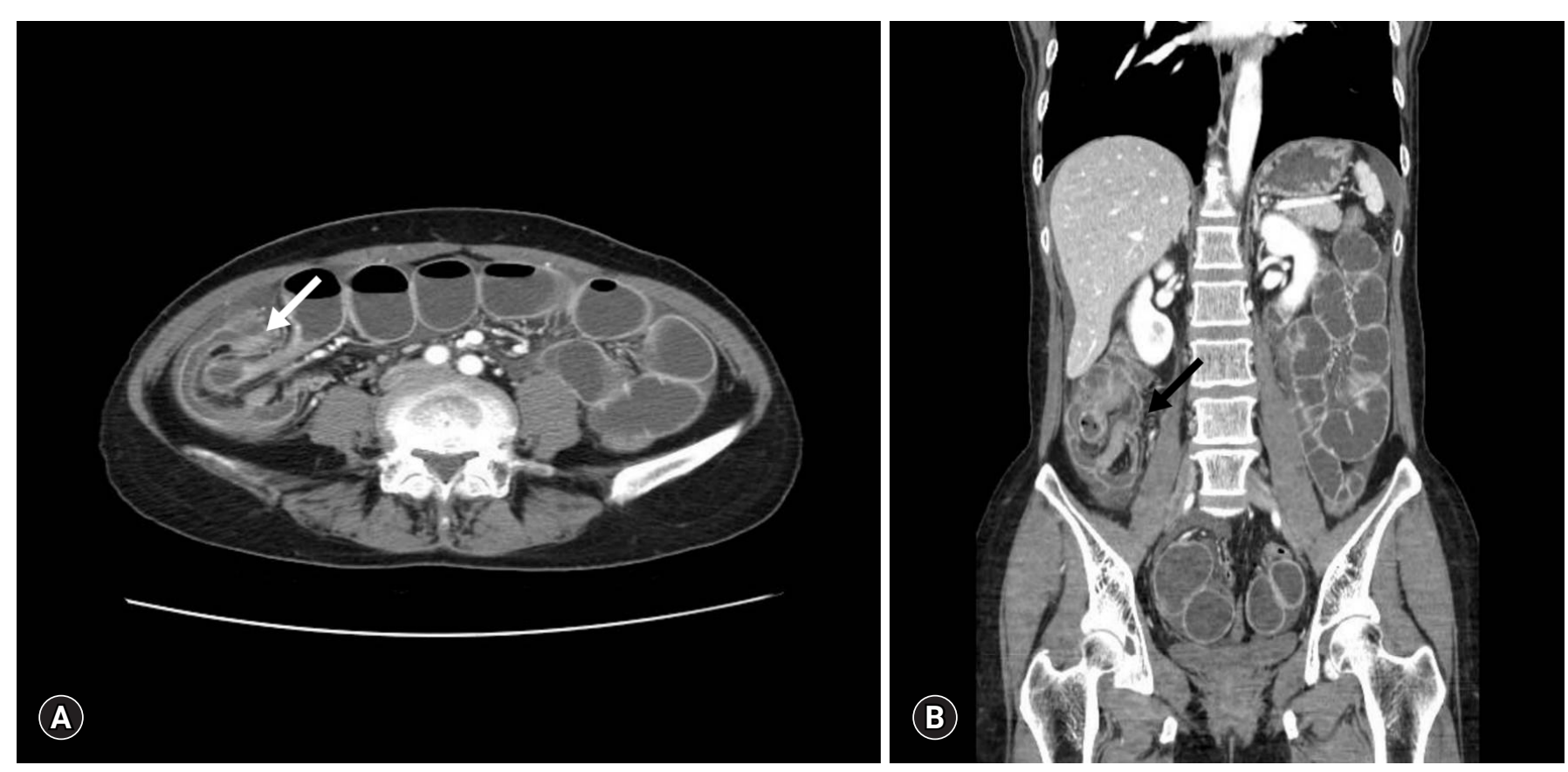

Fig. 1. Abdominal computed tomography scan shows ileocecal intussusception (arrow) with proximal small bowel obstruction. (A) Axial view. (B) Coronal view.
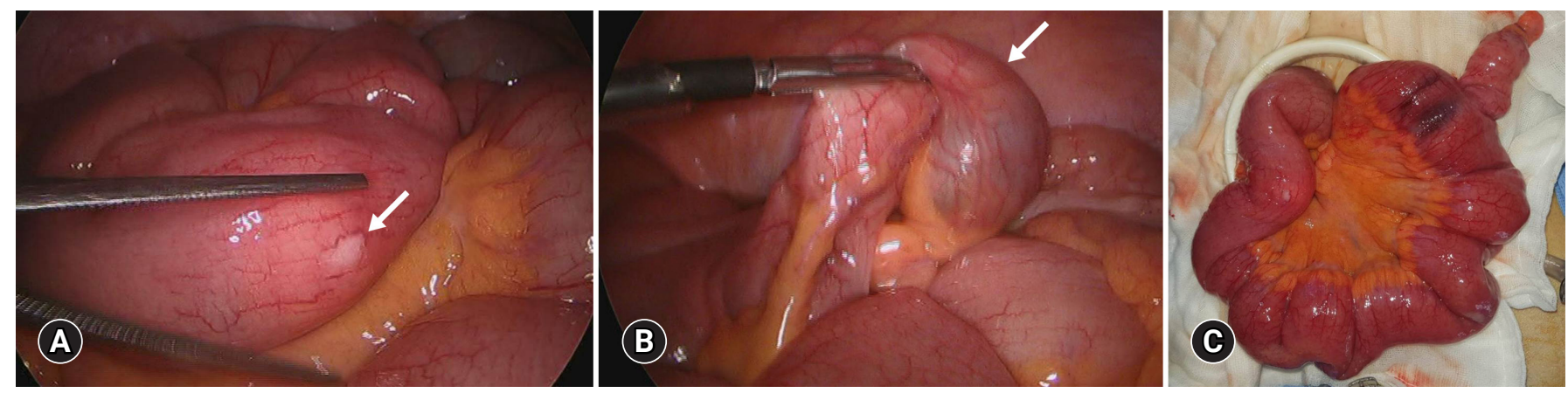

Fig. 2. Intraoperative laparoscopic images. (A) An intraluminal mass (arrow) is observed in the distal ileum approximately $30 \mathrm{~cm}$ from the ileocecal valve. (B) An outpouching closed luminal structure (arrow) is observed on the anti-mesenteric side wall of the ileum, located approximately $70 \mathrm{~cm}$ from the ileocecal valve. (C) The affected ileum is retracted via mini-laparotomy.

men), which revealed spontaneous resolution of intussusception. However, an intraluminal mass was found in the distal ileum, located approximately $30 \mathrm{~cm}$ from the ileocecal valve (Fig. 2A). Further bowel exploration revealed an outpouching closed luminal structure on the antimesenteric side wall of the ileum, located approximately $70 \mathrm{~cm}$ from the ileocecal valve (Fig. 2B). Umbilical mini-laparotomy, approximately $4 \mathrm{~cm}$ in length including the supraumbilical port site, was performed, and the affected ileum was retracted (Fig. 2C). The intraluminal tumor was resected via enterotomy followed by primary closure. The ileal outpouching was resected, and ileal side-to-side anastomosis was performed. The total operative time and estimated blood loss were 90 minutes and $20 \mathrm{~mL}$, respectively.
The patient was discharged on the 4th postoperative day following an uneventful recovery. Histopathological examination confirmed an IFP measuring $2.5 \times 1.5 \mathrm{~cm}$ (Fig. 3) as well as $\mathrm{MD}$ with ectopic pancreatic tissues measuring $5.5 \times 2.5 \mathrm{~cm}$ (Fig. 4).

\section{Discussion}

IFP is a benign gastrointestinal tract lesion and usually asymptomatic. It develops in the submucosa and is composed of mononuclear spindle-shaped cells and prominent eosinophils $[1,3]$. However, small bowel IFPs tend to be symptomatic and present with bleeding or abdominal pain secondary to bowel obstruction, but less commonly occur as compared with gastric IFP [5-7]. Al- 

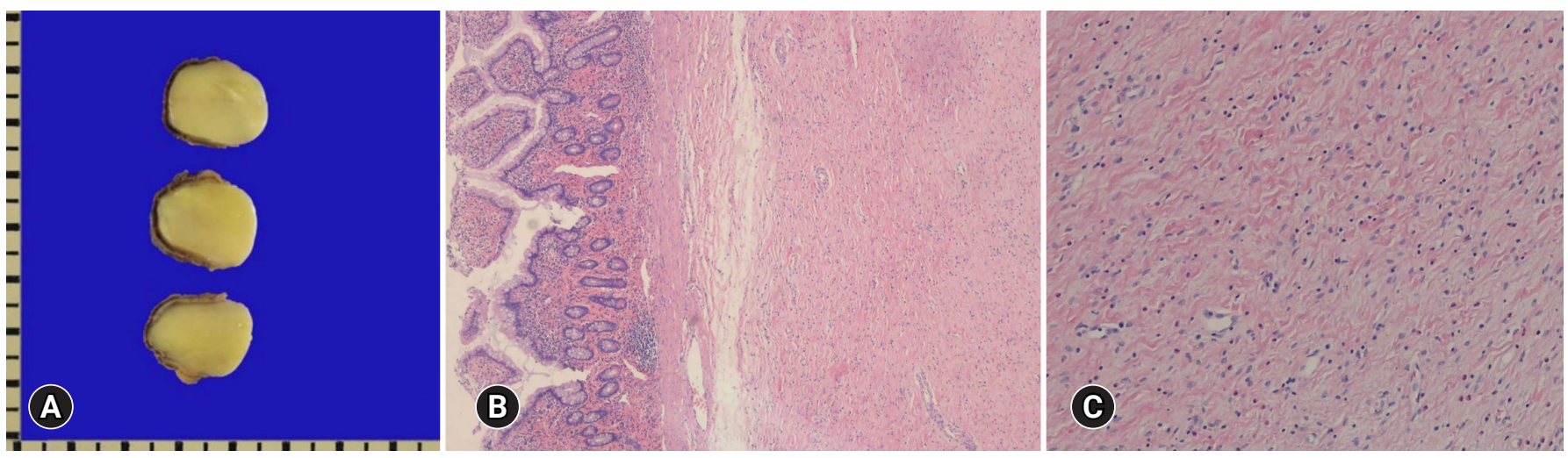

Fig. 3. Gross and microscopic findings of inflammatory fibroid polyp. (A) A well-demarcated submucosal mass is present. (B) It shows loose myxoid fibrous tissue and (C) proliferation of oval to spindle-shaped cell and scattered eosi nophils (hematoxylin and esoin stain, $\times 40[B]$ and $\times 100[C])$.
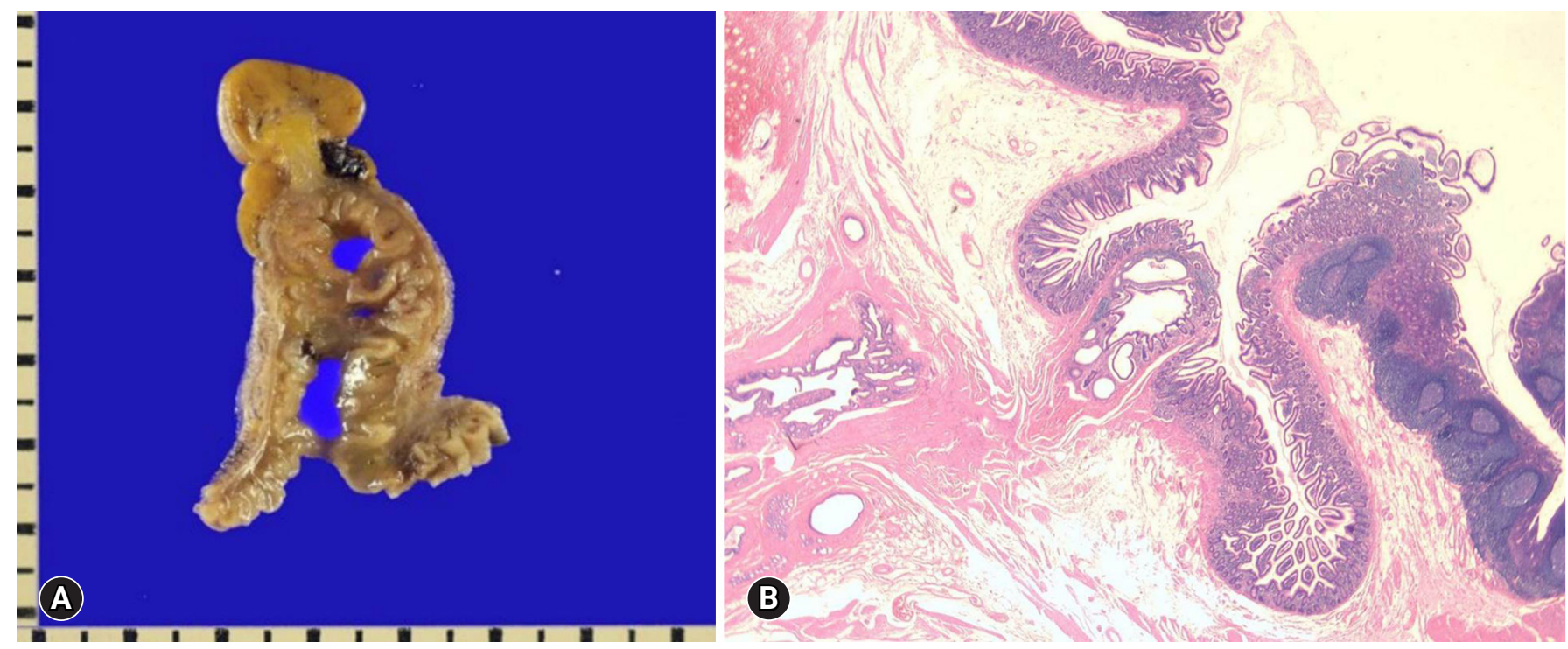

Fig. 4. Gross and microscopic findings of Meckel's diverticulum. (A) Meckel's diverticulum with an outpouching, closed luminal structure is present in the distal ileum. (B) It shows small intestinal mucosal lining with submucosal pancreatic tissue (hematoxylin and eosin stain, $\times 10$ ).

though MD is usually asymptomatic in adults, abdominal pain secondary to obstruction, stricture, or MD-induced intussusception is the predominant characteristic of symptomatic cases [3].

The incidence of adult intussusception secondary to IFP and MD is extremely low. Notably, $<100$ cases of intussusception secondary to ileal IFP and $4 \%-14 \%$ of adult intussusception attributed to intussusception are reported in the existing literature $[1,8,9]$. Moreover, to our knowledge, no report has described the synchronous occurrence of IFP and MD discovered during a surgery for adult intussusception to date.

Intussusception is usually diagnosed by imaging studies because its clinical manifestations and history were nonspecific. CT is preferred over ultrasonography for the differential diagnosis of intussusception because adult intussusception usually present with tumors (including malignancies). In our case, ileocecal intussusception was diagnosed with CT. However, because of the overlapping ileocolic wall, as well as edematous and dilated small bowel loops, CT did not reveal any apparent lesions that might have served as a leading point in this case.

Surgical resection is the primary treatment for adult intussusception, and some patients require radical surgery due to the malignant potential of the tumor within the leading point [10]. Laparoscopic surgery for adult intussusception has been increasingly performed in recent years due to its proven advantages such as faster recovery, less pain, and minimal scarring [10-12]. In addition, laparoscopic exploration is effective even for the confirmative diagnosis of adult intussusception to avoid unnecessary incision.

Therefore, diagnostic laparoscopy was performed first, because 
no pathologic data has confirmed whether the tumor was malignant or benign. In addition, a leading point that caused the ileocolic intussusception was not identified in radiologic examinations. During the laparoscopic exploration, a small ileal mass and MD were detected. However, we could not determine whether one of them was the leading point of intussusception because the ileocolic intussusception resolved spontaneously. However, surgical resection of the two abdominal lesions was performed because both of them were considered as leading points of intussusception.

In conclusion, we report a rare case of synchronous occurrence of ileal IFP and MD detected during laparoscopic surgery for adult intussusception, which was successfully treated with a laparoscopic approach.

\section{Acknowledgments}

\section{Conflicts of interest}

No potential conflict of interest to this article was reported.

\section{ORCID}

Sung Il Kang, https://orcid.org/0000-0002-4751-5779

Mi Jin Gu, https://orcid.org/0000-0002-8350-3038

\section{References}

1. Akbulut S. Intussusception due to inflammatory fibroid polyp: a case report and comprehensive literature review. World J Gastroenterol 2012;18:5745-52.

2. Yakan S, Caliskan C, Makay O, Denecli AG, Korkut MA. Intussusception in adults: clinical characteristics, diagnosis and operative strategies. World J Gastroenterol 2009; 15:1985-9.

3. Unal Kocabey D, Cakir E, Dirilenoglu F, Bolat Kucukzeybek B, Ekinci N, Akder Sari A. Analysis of clinical and pathological findings in inflammatory fibroid polyps of the gastrointestinal system: a series of 69 cases. Ann Diagn Pathol 2018;37:47-50.

4. Lequet J, Menahem B, Alves A, Fohlen A, Mulliri A. Meckel's diverticulum in the adult. J Visc Surg 2017;154:253-9.

5. Liu TC, Lin MT, Montgomery EA, Singhi AD. Inflammatory fibroid polyps of the gastrointestinal tract spectrum of clinical, morphologic, and immunohistochemistry features. Am J Surg Pathol 2013;37:586-92.

6. Kimura N, Hight M, Liang J, Willy R, Liang K, Camp J. Adult intussusception secondary to inflammatory fibroid polyp. West J Emerg Med 2015;16:581-2.

7. Mader S, Ting J, Nabi H. Ileocolic intussusception from an inflammatory fibroid polyp: a rare cause of adult small bowel obstruction. ANZ J Surg 2019;89:E100-1.

8. Moore T, Johnston AO. Complications of Meckel's diverticulum. Br J Surg 1976;63:453-4.

9. Ymaguchi M, Takeuchi S, Awazu S. Meckel's diverticulum: investigation of 600 patients in Japanese literature. Am J Surg 1978; 136:247-9.

10. Kang SI, Kang J, Kim MJ, Kim IK, Lee J, Lee KY, et al. Laparoscopic-assisted resection of jejunojejunal intussusception caused by a juvenile polyp in an adult. Case Rep Surg 2014; 2014:856765.

11. Paya Llorente C, Martinez Perez A, Bernal Sprekelsen JC, Sebastian Tomas JC, Armananzas Villena E. Laparoscopic surgery for adult enterocolic intussusception: case report and literature review. Gastroenterol Hepatol 2018;41:255-7.

12. Namikawa T, Okamoto K, Okabayashi T, Kumon M, Kobayashi M, Hanazaki K. Adult intussusception with cecal adenocarcinoma: successful treatment by laparoscopy-assisted surgery following preoperative reduction. World J Gastrointest Surg 2012;4:131-4. 